\title{
Nostalgie 2.0
}

\author{
Damien Hallegatte ${ }^{\mathrm{a}}$
}

RÉSUMÉ. Les 30 dernières années de recherches sur la nostalgie, en marketing d'abord et en psychologie ensuite, ont considérablement fait avancer les connaissances sur cette émotion fascinante qui se trouve au cœur de nos vies personnelles, et dorénavant de notre vie collective. Cependant, cinq éléments se doivent encore d'être éclaircis : la nature même de la nostalgie comme émotion liée à un passé heureux et non comme préférence pour les choses du passé; la différence entre l'ancienneté perçue d'un objet et la nostalgie éventuelle d'un individu pour l'objet ancien; la distinction entre la propension à la nostalgie et la croyance au déclin; la distinction entre les dimensions spécifique et globale de la croyance au déclin; ainsi que le lien entre l'âge et la nostalgie. Cet article propose des raffinements et des changements conceptuels sur les quatre premiers éléments, grâce à des arguments théoriques et deux études quantitatives, ainsi que l'examen du lien entre l'âge et la nostalgie. Avant cela, nous offrons un résumé survolant plus de 300 ans d'histoire du concept de nostalgie. Les résultats de cette recherche nous amènent à conclure que l'expression « la nostalgie n'est plus ce qu'elle était » n'est pas un simple aphorisme, mais qu'elle décrit la réalité de ce concept au $21^{\mathrm{e}}$ siècle.

\begin{abstract}
Knowledge on nostalgia, this fascinating emotion that we may encounter personally or even collectively on a daily basis, accumulated rapidly in the last 30 years of research, in marketing first and then in psychology. However, five concerns still remain: the nature of nostalgia as an emotion triggered by evocation of the past and not as a preference for things of the past; the distinction between perceived age of an object and nostalgia felt for that object; the distinction between nostalgia proneness and belief in decline; the distinction between the specific and the global dimension of belief in decline; the link between age and nostalgia. This article propose conceptual refinements and changes relative to the four first concerns, by theoretical arguments and two quantitative study, and the evaluation of the link between age and nostalgia, as well as a very short history of the concept of nostalgia. To conclude, we may say that the expression "nostalgia is not what is used to be" is not simply an aphorism, but describe the reality of that concept in the $21^{\text {st }}$ century.
\end{abstract}

\section{Introduction}

La nostalgie intéresse les chercheurs en marketing depuis le début des années 1990. Les articles séminaux de Belk (1990), de Havlena et Holak (1991) et de Holbrook et Schindler (1991) se sont intéressés aux premières publicités faisant appel à la nostalgie de la fin des années 1980, où la chute du communisme, l'arrivée des premiers bébé-boumeurs dans la quarantaine, et l'imminence de la fin du siècle, entre autres, ont modifié le rapport des sociétés occidentales à leur passé récent. Tout au cours des années 1990, la discipline du marketing a réhabilité la nostalgie comme émotion ambivalente, donc partiellement positive, suivi de la psychologie la décennie suivante, qui a mis en évidence ses effets positifs sur le bien-être psychologique. Parallèlement, une série d'études a soutenu empiriquement

a Professeur, Ph. D., Université du Québec à Chicoutimi l'efficacité de la suscitation de la nostalgie comme stratégie de création publicitaire, validant scientifiquement les pratiques de l'industrie.

Cependant, malgré les avancées majeures des connaissances sur la nostalgie des presque 30 dernières années, plusieurs éléments conceptuels sur la nature même de la nostalgie, et certaines de ses propriétés, demeurent confus dans la littérature scientifique en marketing. Le plus gros problème, et la source directe ou indirecte de tous les autres problèmes conceptuels traités dans cet article, se trouve dans la définition de la nostalgie de Holbrook et Schindler (1991), selon laquelle il s'agit d'une préférence pour le passé. En effet, encore aujourd'hui, elle demeure une référence en marketing, alors qu'elle est en contradiction avec toutes les 
autres définitions élaborées en marketing et en psychologie, lesquelles considèrent la nostalgie comme une émotion, un sentiment ou une humeur (Hallegatte et Marticotte, 2014), qui pourrait être liée à un passé positif (Godbole, Shehryar et Hunt, 2006) c'est-à-dire qui pourrait être le symptôme d'un passé heureux.

De ce problème originel en découle trois autres. Premièrement, le qualificatif " nostalgique » est couramment utilisé en tant que synonyme d'« ancien » comme dans l'expression " marque nostalgique » ou en tant que raccourci de la locution « faisant appel à la nostalgie » comme dans l'expression "publicité nostalgique ", alors que le simple bon sens permet de distinguer la nostalgie ressentie par un individu pour un objet de l'ancienneté perçue de l'objet. Deuxièmement, le concept de propension à la nostalgie est amalgamé avec celui de croyance au déclin, amalgame entériné plus d'une décennie plus tard par les auteurs mêmes de la définition problématique (Schindler et Holbrook, 2003). Troisièmement, la propension à la nostalgie telle que mesurée par l'index de nostalgie de Holbrook $(1993,1994)$ serait bidimensionnelle, alors qu'il n'y a aucune raison théorique de penser cela. Par ailleurs, il existe une autre zone obscure, qui n'est pas directement reliée à la définition de Holbrook et Schindler (1991), et qui concerne le lien ou l'absence de lien entre l'âge et la propension à la nostalgie. L'objectif de cet article est de clarifier ces cinq éléments, grâce à deux études quantitatives pour quatre d'entre eux, non sans avoir effectué un bref historique du concept fascinant de nostalgie.

\section{La nostalgie, de la médecine à la psychologie, en passant par le marketing}

La première conceptualisation de la nostalgie, et l'invention du terme lui-même sont attribuées à Johannes Hofer (1669-1752), un médecin suisse qui a observé des pathologies parmi les militaires en mission à l'étranger, causées par l'éloignement de leur mère patrie. Hofer s'intéressait à cette maladie liée à un déplacement géographique, qui était désignée dans le langage courant comme «la maladie du pays ", par les Français, ou " heimweh », par les Helvètes (Hofer, 1688). Hofer avait besoin d'un terme savant pour nommer cette maladie : il créa le mot nostalgie, composé du grec nostos, qui signifie «le retour », et algos, qui signifie " la souffrance ». Il intitula sa thèse de doctorat en philosophie et médecine, présentée en 1688 à l'Université de Bâle (Suisse), "Thèse de médecine sur la nostalgie ou le mal du pays », afin de clarifier ce néologisme. Cette maladie était composée de symptômes aussi divers que l'obsession du pays natal, la tristesse chronique, les troubles du sommeil, la mélancolie, la fièvre ou des palpitations cardiaques (Hofer, 1688). Des purges, des saignements ou l'absorption de certaines mixtures étaient préconisés, mais il s'agissait seulement de palliatifs permettant d'améliorer l'état du patient afin qu'il supporte un voyage. Le retour au pays constituait le remède le plus rapide et le plus efficace contre cette maladie (Hofer, 1688).

Au 18 siècle, le concept de nostalgie s'éloigne de la notion de «mal du pays » et intègre la dimension temporelle que l'on connait aujourd'hui. Rousseau et Kant sont parmi les premiers à proposer une définition moderne de la nostalgie (Divard et Robert-Demontrond, 1997), qu'ils considèrent comme un regret du passé. Aux 19e et 20e siècles, les dimensions temporelle et spatiale de la nostalgie vont cohabiter. En médecine et en psychologie, la nostalgie restera encore longtemps associée au mal du pays, les deux termes étant généralement utilisés comme des synonymes, au moins jusqu'au milieu du 20e siècle (voir Martin, 1954; McCann, 1941). De plus, la psychologie considérera la nostalgie comme un désordre psychologique pendant encore la majorité du 20e siècle (Wildschut et al., 2006). D'autres domaines, comme la littérature, commenceront à considérer la nostalgie comme une expérience humaine normale reliée au passé, et non comme une maladie, bien avant les sciences sociales, comme dans le roman $\grave{A}$ la recherche $d u$ temps perdu de Marcel Proust.

Après avoir été abordée en littérature, pensée par des philosophes, conceptualisée en médecine et en psychologie, la nostalgie a été réinterprétée en sociologie. Dans un ouvrage qui fait figure de référence, Davis (1979) expurge définitivement la nostalgie de son aspect morbide et scelle son émancipation conceptuelle par rapport au mal du pays, dont elle est issue. Dès lors, il y aura un large consensus dans la littérature scientifique sur ces deux aspects fondamentaux de la nostalgie: il s'agit d'une réaction humaine normale et non une maladie, qui est reliée au passé et non à un lieu. 
Le concept de nostalgie a ensuite été étudié en marketing à partir du début des années 1990. S'appuyant sur les travaux de Fred Davis (1979), la nostalgie est considérée, dès le début des recherches en marketing, comme une émotion ambivalente, de type douce-amère (Belk, 1990, 1991; Havlena et Holak, 1991). En effet, la nostalgie implique en même temps des souvenirs agréables et la conscience d'une perte, du caractère inaccessible d'un passé idéalisé. La complexité émotive de la nostalgie est mise en évidence par Holak et Havlena (1998), qui montrent que la nostalgie est associée en même temps à des émotions positives comme la joie, l'affection et la gratitude, et à des émotions négatives ou mitigées comme la tristesse et le désir.

Après avoir caractérisé la nostalgie, la recherche en marketing s'est intéressée aux effets de la nostalgie ressentie à la suite de l'exposition à une publicité dite nostalgique, c'est-à-dire qui utilise des éléments (personnes, objets, expériences, idées, etc.) associés à un passé plus ou moins distant, généralement idéalisé et qui suscite la nostalgie. Ces recherches montrent clairement que susciter la nostalgie a un effet positif sur les réponses des consommateurs, que ce soit sur l'attitude envers la publicité (p. ex. : Muehling et Pascal, 2011), l'attitude envers la marque (p. ex. : Marchegiani et Phau, 2011) l'attitude envers le produit (p. ex. : Bambauer-Sachse et Gierl, 2009) ainsi que sur l'intention d'achat (p. ex. : Marchegiani et Phau, 2011). En effet, les publicités qui suscitent la nostalgie créent des émotions plus positives et évoquent des images mentales plus intenses (Bambauer-Sachse et Gierl, 2009) et induisent des niveaux plus élevés de réflexion sur soi et d'implication envers la publicité (Muehling et Pascal, 2012) que les autres publicités. En outre, il a été montré que les «marques nostalgiques » ont un effet positif sur l'humeur des consommateurs, contrairement aux marques " non nostalgiques » (Orth et Gal, 2012).

Parallèlement, un second courant de recherche sur les effets de la nostalgie en marketing s'est intéressé aux différences individuelles en matière de prédisposition à ressentir de la nostalgie, qui serait susceptible d'influencer, de manière relativement stable, les préférences pour des produits associés au passé, voire d'autres types de produits. Holbrook $(1993,1994)$ propose que certains individus soient plus prompts à ressentir de la nostalgie que d'autres

Revue Organisations \& Territoires • Volume 26・ N 1 1-2 2017 et crée une variable psychographique, la " propension à la nostalgie ». L'« attitude envers le passé », qui en serait une mesure (Schindler et Holbrook, 2003) influence les préférences envers les produits du passé (Holbrook, 1993; Holbrook et Schindler, 1994, 1996; Schindler et Holbrook, 2003). Par exemple, les consommateurs dont la propension à la nostalgie est élevée ont tendance à aimer mieux les films sortis aux alentours de la fin de leur adolescence (19 ans) alors que ceux dont la propension à la nostalgie est faible ont tendance à préférer les films sortis aux alentours de la fin de leur vingtaine (28 ans) (Holbrook et Schindler, 1996). Ce même phénomène a été mis en évidence pour les préférences envers les stars de cinéma (Holbrook et Schindler, 1994) ainsi qu'envers les automobiles (Schindler et Holbrook, 2003).

Cependant, l'ensemble de ces études qui utilisent l'index de nostalgie de Holbrook pourrait être invalide (Hallegatte et Marticotte, 2014), à moins de considérer la croyance au déclin comme un indicateur de la propension à la nostalgie (Schindler et Holbrook, 2003), position réfutée théoriquement par Hallegatte et Marticotte (2014) et contredite empiriquement dans la suite de cet article. Plus généralement, la propension à la nostalgie influence positivement l'intention d'achat de produits issus de son passé personnel (Sierra et McQuitty, 2007) ainsi que l'intention de donner à une œuvre de charité (Merchant, Ford et Rose, 2011; Zhou et al., 2012b), et négativement l'usage d'Internet (Reisenwitz et al., 2007).

Après avoir été réhabilitée par la discipline du marketing dans les années 1990, la nostalgie a suscité de l'intérêt en psychologie une quinzaine d'années plus tard, le point de départ de cette récente quête empirique étant Wildschut et al. (2006). Ces recherches ont notamment confirmé que la nostalgie est associée en même temps à la joie et à la tristesse (Barrett et al., 2010). D'autres études précisent que la signature affective de la nostalgie, quoique mitigée, est principalement positive (Hepper et al., 2010; Hepper et al., 2012; Wildschut et al., 2006). En outre, dans les récits analysés par Wildschut et al. (2006), les éléments négatifs étaient combinés avec les éléments positifs pour construire un récit de rédemption, qui progresse du négatif au positif (Wildschut et al., 2006). Dans la nostalgie, le négatif serait au service du positif, en quelque sorte. Notons, de plus, que l'affect positif de la nostalgie pourrait provenir, au moins partiellement, d'une 
erreur d'attribution de l'affect créée par le succès du recouvrement d'informations en mémoire. Dit autrement, l'affect positif serait attribué au contenu du recouvrement, c'est-à-dire au passé lui-même, plutôt qu'au succès du recouvrement, alors que ce dernier en serait la vraie cause (Leboe et Ansons, 2006).

Mais l'apport le plus significatif de la psychologie par rapport au marketing réside dans la découverte de fonctions psychologiques importantes de la nostalgie (p. ex., Iyer et Jetten, 2011; Juhl et al., 2010; Routledge et al., 2008; Routledge et al., 2011; Wildschut et al., 2006; Zhou et al., 2008). D'abord, elle permet d'améliorer l'image de soi, notamment parce qu'elle est associée à des moments importants de la vie des individus, en interaction avec des personnes significatives (Wildschut et al., 2006). De plus, étant un dépositaire de liens sociaux antérieurs, la nostalgie peut servir de stratégie indirecte pour faire face à la solitude (Wildschut et al., 2010). D'ailleurs, les personnes qui sont prédisposées à la nostalgie ont tendance à être orientées vers autrui (Batcho, 1998, 2007). La nostalgie induit l'empathie et les comportements charitables (Zhou et al., 2012b) ainsi que la tolérance envers les autres (Turner, Wildschut et Sedikides, 2012). En outre, la nostalgie agit comme une véritable ressource existentielle : elle aide à donner un sens à sa vie (Routledge et al., 2011) et même à faire face à la conscience de sa propre mort (Juhl et al., 2010; Routledge et al., 2008). Ainsi, la nostalgie constitue une véritable ressource psychologique pour protéger sa santé mentale (Zhou et al., 2008). Ajoutons qu'en plus d'aider à maintenir son confort psychologique, la fonction homéostatique de la nostalgie permettrait de maintenir son confort physiologique (Zhou et al., 2012a).

\section{La nostalgie, symptôme d'un passé heureux}

La plupart des auteurs en marketing s'accordent sur la nature affective de la nostalgie, en la considérant comme une émotion, un sentiment ou une humeur (p. ex., Belk, 1990; Divard et Robert-Demontrond, 1997; Holbrook et Schindler, 1991; Stern, 1992). De même, en psychologie, la quasi-totalité des auteurs considère la nostalgie comme une émotion (Sedikides et al., 2008; Sedikides, Wildschut et Baden, 2004). L'exception notable à ce consensus interdisciplinaire est la définition de Holbrook et Schindler (1991) selon laquelle la nostalgie est « une préférence (un penchant, une attitude positive ou un affect favorable) envers des objets (personnes, lieux ou choses) qui étaient plus communs (populaires, à la mode ou largement disponibles) lorsque l'individu était plus jeune (au début de l'âge adulte, dans l'adolescence, dans l'enfance, et même avant la naissance $)^{1} »$. Cette définition fait encore référence aujourd'hui dans le domaine du marketing, probablement par le caractère pionnier de l'écrit en question, le statut de démiurge de l'un des deux auteurs, et le pragmatisme managérial qui s'en dégage. Cependant, nous pensons que cette définition de la nostalgie amalgame la cause (la nostalgie) et la conséquence (les préférences). Il nous paraît clair que préférer des produits qui étaient plus communs lorsque l'individu était plus jeune n'est pas la même chose qu'être nostalgique, quand bien même les deux choses seraient fréquemment liées.

La nostalgie peut certes médiatiser ou modérer la relation entre l'ancienneté perçue d'un produit et la préférence pour celui-ci. Cependant, nous pensons que l'on peut être nostalgique sans préférer des produits anciens, mais plutôt des produits modernes, car ils sont plus fonctionnels, performants et d'esthétique contemporaine. Parallèlement, on peut préférer des produits anciens sans être nostalgique, mais parce qu'ils sont plus robustes, qu'ils ne sont pas frappés d'une odieuse obsolescence programmée, ou parce qu'ils nous sont familiers. En revanche, la définition de la nostalgie de Holbrook et Schindler (1991) apparaît comme étant teintée de l'idée selon laquelle le passé et le présent sont opposés et que, par conséquent, être nostalgique équivaut à préférer les choses du passé par rapport à celles du présent, conformément à Davis (1979) qui considère que la nostalgie est associée non seulement à un attrait du passé, mais aussi à un rejet du présent et probablement du futur.

Or, selon Zimbardo et Boyd (1999), un individu peut être en même temps orienté vers le passé et le futur, et le bien-être psychologique pourrait être relié à une orientation temporelle équilibrée entre le passé, le présent et le futur. L'orientation vers le futur permet aux gens d'atteindre leurs objectifs, l'orientation vers le passé permet de rester connecté avec ses racines et sert de base à son identité personnelle, et l'orientation vers le présent permet de profiter des plaisirs de la vie (Zimbardo et Boyd, 1999). L'idée que l'orientation vers le passé n'est pas opposée avec une orientation vers le futur et 
une orientation vers le présent est aussi soutenue dans l'étude de Spears et Amos (2012). D'ailleurs, les personnes fortement nostalgiques évaluent le monde lorsqu'ils étaient jeunes plus favorablement que les personnes faiblement nostalgiques, mais leur évaluation du monde présent et du monde tel qu'ils le perçoivent dans le futur (20 ans plus tard) ne varie pas de manière significative (Batcho, 1995). Ces résultats, confirmés dans une étude subséquente (Batcho, 1998), indiquent que la nostalgie n'implique pas forcément un rejet du présent ou une forme de pessimisme (Batcho, 1995). Une autre étude montre que les perspectives sur le futur n'ont pas d'effet direct sur la nostalgie (Godbole, Shehryar et Hunt, 2006). L'idée selon laquelle la nostalgie n'est pas nécessairement reliée à une attitude négative envers le présent et le futur est cohérente avec la conceptualisation actuelle de la nostalgie, vue comme une émotion non seulement principalement positive, mais qui contribue au bien-être psychologique (p. ex., Iyer et Jetten, 2011; Juhl et al., 2010; Routledge et al., 2008; Routledge et al., 2011; Sedikides et al., 2008; Sedikides, Wildschut et Baden, 2004; Wildschut et al., 2006; Wildschut et al., 2010; Zhou et al., 2008).

Nous proposons donc, contrairement à Holbrook (1993, 1994), qui considère que la nostalgie est une préférence pour le passé et un rejet ou une attitude négative envers le présent et le futur, de distinguer quatre concepts : la nostalgie; l'attitude envers le passé; l'attitude envers le présent; l'attitude envers le futur. En particulier, une attitude négative envers le futur, plutôt que d'être une dimension de la propension à la nostalgie, s'apparente plutôt à du pessimisme. En outre, une attitude négative envers le présent s'apparente plutôt à une insatisfaction relative à ses conditions de vie qu'à de la nostalgie. Ainsi, plutôt que d'être, par nature, une préférence pour le passé, qui implique une comparaison entre le passé, le présent et le futur, où le passé est perçu comme supérieur, il est probable que la nostalgie soit plutôt reliée à un passé personnel positif, nonobstant les conditions actuelles ou les projections dans le futur. En effet, le simple bon sens indique que l'on ne peut être nostalgique de notre passé que si celui-ci a été heureux.

Compte tenu de ce qui précède, nous posons l'hypothèse suivante :

$\mathrm{H}_{1}$ : La nostalgie est reliée à un passé positif.

\section{L'ancienneté d'un objet et la nostalgie d'un individu}

L'importance de la nostalgie en marketing et la richesse sémantique du terme sont telles que les chercheurs ont créé des expressions comme "produit nostalgique » (Loveland, Smeesters et Mandel, 2009, 2010), " marque nostalgique » (Kessous, Roux et Chandon, 2015; Orth et Gal, 2012), « achat nostalgique » (Sierra et McQuitty, 2007), « préférence nostalgique » (Holbrook, 1993), " publicité nostalgique » (Bambauer-Sachse et Gierl, 2009; Muehling, Sprott et Sultan, 2014; Zhao, Muehling et Kareklas, 2014), " technique nostalgique » (Reisenwitz, Iyer et Cutler, 2004), " attachement nostalgique » (Schindler et Holbrook, 2003) et «associations nostalgiques » (Cattaneo et Guerini, 2012). Mais, de notre point de vue, qu'une préférence ou un produit soient, par exemple, qualifiés de nostalgique est une métonymie semant la confusion, voire un abus de langage. Pire encore, faire équivaloir « ancien » avec « nostalgique » amalgame le stimulus (l'objet ancien) avec la réponse potentielle (la nostalgie). Une préférence ne peut pas être nostalgique, un produit ne peut pas être nostalgique et une marque ne peut pas être nostalgique. Seul un être humain peut être nostalgique. Nous préférons qualifier une marque populaire actuellement et qui l'était dans le passé de marque classique ou de marque familière. Une marque classique est intemporelle, alors que la nostalgie implique une temporalité, une réaction à un stimulus associé au passé. Une marque ancienne peut être classique si elle jouit encore aujourd'hui d'une notoriété ou d'une image favorable; sinon, il s'agit d'une marque en déclin. Et une marque ancienne est une marque rétro si elle est associée de manière relativement explicite au passé (Hallegatte, 2014).

Outre le caractère discutable du terme "produit nostalgique ", son opérationnalisation apparait problématique, révélant encore davantage les problèmes conceptuels. Dans une étude, les marques (ou produits) nostalgiques choisies comme stimuli sont « des marques qui étaient populaires dans le passé (et qui sont toujours populaires aujourd'hui)» alors que les marques non nostalgiques sont « des marques qui sont populaires aujourd'hui (mais qui l'étaient moins dans le passé ou qui n'existaient pas dans le passé » (Loveland, Smeesters et Mandel, 2010, p. 397). La différence entre ces deux types de marque n'est pas leur niveau de popularité actuel, 
qui est identique, mais leur niveau de popularité passé (plus faible ou inexistant dans le cas des marques non nostalgiques). Or, un objet de nostalgie, selon Holbrook et Schindler (1991), est un objet qui était « plus commun (populaire, à la mode ou largement disponible) lorsque l'individu était plus jeune » et donc qui, « pour quelque raison que ce soit, n'est plus expérimenté couramment aujourd'hui ».

Malgré les faiblesses majeures de la définition de Holbrook et Schindler (1991), il apparait effectivement logique que l'on ne puisse pas être réellement nostalgique de quelque chose qui est toujours aussi populaire ou disponible que dans le passé, comme des biscuits que l'on mangeait lorsque l'on était enfant, mais que l'on retrouve encore aujourd'hui dans toutes les épiceries. En effet, la nostalgie implique un manque, une perte, une relative inaccessibilité de l'objet de nostalgie. Dit autrement, si on est fréquemment exposé à un stimulus que l'on associe au passé, il est probable qu'on l'associe de moins en moins au passé, et qu'il ait moins de chance de susciter la nostalgie, comme le groupe de rock The Rolling Stones, par exemple, de retour depuis plus de 20 ans. Donc, l'opérationnalisation du concept de marque nostalgique proposée par Loveland et al. (2010) est en contradiction avec son origine conceptuelle, la définition de la nostalgie de Holbrook et Schindler (1991). Plus globalement, la marque Coca-Cola, par exemple, est une « marque qui était populaire dans le passé (et qui est toujours populaire aujourd'hui) ", mais peu la qualifieraient de

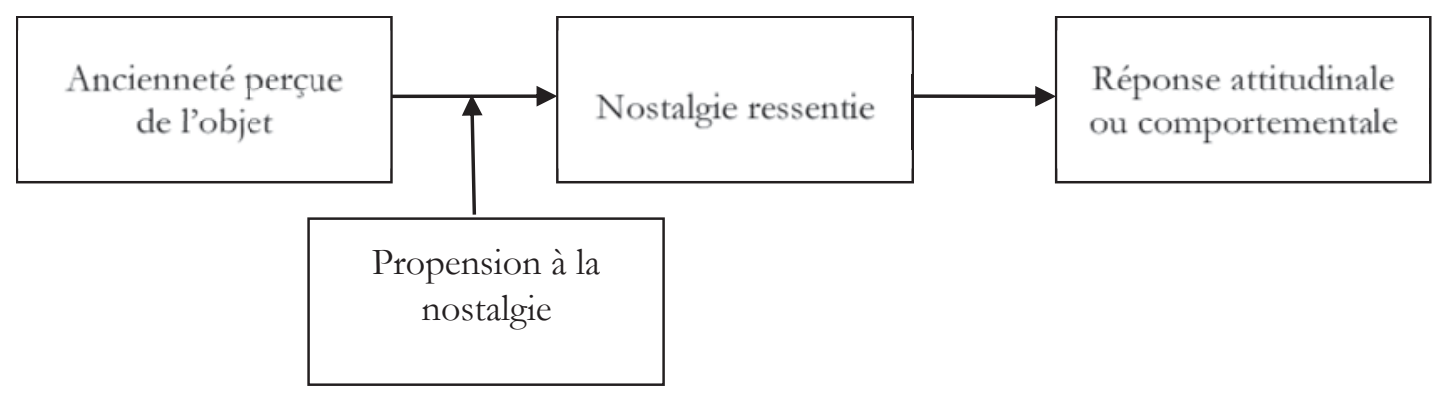

Figure 1 - Distinction entre la nostalgie et l'ancienneté perçue d'un objet

\section{La propension à la nostalgie et la croyance au déclin}

En lien avec la définition de la nostalgie de Holbrook et Schindler (1991), qui fait toujours référence en marketing, l'index de nostalgie de Holbrook $(1993,1994)$
« marque nostalgique ». L'opérationnalisation du concept de marque nostalgique de Loveland et al. (2010) est donc aussi en contradiction avec le bon sens.

Ainsi, « ancien » n'est pas synonyme de " nostalgique », la nostalgie étant réservée aux humains, et préférer un produit du passé n'équivaut pas à être nostalgique. Une marque ou un produit familier pour un consommateur peut constituer un « vieux favori » (couramment appelé, pour les produits agroalimentaires, «comfort food»), mais la préférence dont elle est l'objet n'est nullement reliée à la nostalgie (Wood, 2010). Nous proposons donc de distinguer, d'une part, les concepts d'ancienneté perçue d'un objet, qui est une interprétation d'un ensemble de stimuli, de la nostalgie ressentie, qui en est une réponse émotionnelle possible. Nous utilisons le terme «nostalgie ressentie » ici afin d'effectuer une distinction claire avec la propension à la nostalgie. D'autre part, nous proposons de distinguer la nostalgie ressentie de sa réponse attitudinale ou comportementale potentielle (p. ex. : préférence). Ainsi, dans le réseau nomologique suivant (voir figure 1), la nostalgie ressentie est vue comme un mécanisme possible par lequel un objet perçu comme ancien cause une réponse attitudinale ou comportementale. Dit autrement, la nostalgie ressentie serait une variable médiatrice de la relation entre l'ancienneté perçue de l'objet et la réponse attitudinale ou comportementale. La propension à la nostalgie, quant à elle, est une variable modératrice potentielle de la relation entre l'ancienneté perçue de l'objet de la réponse attitudinale ou comportementale (voir figure). est utilisé dans la quasi-totalité des recherches en marketing qui traitent de la propension à la nostalgie. Cependant, il n'est pas sûr que cette échelle mesure la propension à la nostalgie per se. En fait, cette échelle de mesure semble invalide (Hallegatte et Marticotte, 2014). Après sa création, la propension à la nostalgie 
sera rapidement appelée aussi « attitude envers le passé » (Holbrook et Schindler, 1994) et, par la suite, les deux termes seront utilisés comme synonymes (Holbrook et Schindler, 1996; Schindler et Holbrook, 2003). Parmi ces deux dénominations, « attitude envers le passé » apparait être celle représentant mieux le construit mesuré par l'index de nostalgie. En effet, les énoncés ont été élaborés pour représenter l'idée selon laquelle le passé était meilleur, les choses étaient mieux avant qu'aujourd'hui (Holbrook, 1993, 1994), conformément à la conceptualisation de la nostalgie de Davis (1979), à partir de laquelle cette échelle a été développée, conceptualisation que nous remettons en question dans cet article. Mais, en outre, cette échelle mesure non seulement l'attitude envers le passé (p. ex. : «Les produits sont de plus en plus de mauvaise qualité »), mais aussi l'attitude envers le présent (p. ex. : " Le niveau de vie actuel est le plus élevé jamais atteint ») et l'attitude envers le futur (p. ex. : «Dans le futur, les personnes auront une vie encore meilleure »), dont les échelles sont bien évidemment inversées. Ainsi, les énoncés de cette échelle reflètent l'idée selon laquelle la nostalgie ne représente pas seulement un attrait pour le passé, mais aussi une insatisfaction envers le présent (Davis, 1979) et une inquiétude relative au futur (Davis, 1979; Nawas et Platt, 1965), conformément à la définition de la nostalgie de Holbrook et Schindler (1991), comme discuté précédemment.

La validité de cet index de nostalgie de Holbrook $(1993,1994)$ a été directement remise en question par Hallegatte et Marticotte (2014), puisqu'elle partage les faiblesses conceptuelles de la définition de la nostalgie de Holbrook et Schindler (1991). Les auteurs proposent que l'index de nostalgie de Holbrook mesure en fait la croyance au déclin, tel qu'implicitement reconnu par l'auteur de l'échelle lui-même (Schindler et Holbrook, 2003). Pour soutenir leurs arguments théoriques, Hallegatte et Marticotte (2014) indiquent que la croyance au déclin est indépendante de l'attrait pour les choses du passé, comme mesuré par des échelles issues de la psychologie environnementale (Schindler et Holbrook, 2003). En outre, l'index de nostalgie de Holbrook $(1993,1994)$ n’est pas corrélé avec l'inventaire nostalgique de Batcho (1995) (Batcho, 2007; Batcho et al., 2008), qui est une échelle établie en psychologie (Routledge et al., 2008). Cependant, les auteurs ne fournissent aucune donnée empirique pour soutenir directement leur démonstration théorique, selon laquelle la croyance au déclin ne peut

Revue Organisations \& Territoires • Volume 26・ No 1-2 • 2017 être considérée comme un indicateur de la propension à la nostalgie.

Or, il existe une échelle de mesure de la propension à la nostalgie dans la littérature, la Southampton Nostalgia Scale (SNS) (Routledge et al., 2008), qui mesure principalement la fréquence à laquelle un individu ressent de la nostalgie, et constitue ainsi une mesure directe de la propension à la nostalgie. A contrario, l'inventaire nostalgique de Batcho (1995) permet de déterminer à quel point certains éléments du passé manquent aux répondants (jouets, émissions télévisées, insouciance, la société telle qu'elle était), mais ne mesure ainsi qu'un aspect de la nostalgie qui est apparue par la suite comme étant une émotion complexe (Wildschut et al., 2006). Néanmoins, il est notable que la SNS soit corrélée avec cet inventaire nostalgique de Batcho (1995), mais aussi un sous-ensemble de huit énoncés du Zimbardo Time Perspective Inventory (ZTPI) (Zimbardo et Boyd, 1999) utilisé par Routledge et al. (2008) comme mesure d'attitude envers le passé.

Compte tenu de ce qui précède, et afin de valider la conclusion de Hallegatte et Marticotte (2014) selon laquelle la propension à la nostalgie et la croyance au déclin sont des concepts distincts, nous posons l'hypothèse suivante :

$\mathrm{H}_{2}$ : La propension à la nostalgie et la croyance au déclin sont des concepts distincts.

\section{La croyance au déclin spécifique et global}

Partant de l'idée que l'index de nostalgie de Holbrook $(1993,1994)$ mesure la croyance au déclin (Hallegatte et Marticotte, 2014), un construit pertinent en marketing, nous nous sommes intéressé à ses propriétés psychométriques, qui sont apparues problématiques. D'abord, la fidélité de cette échelle est variable. Bien que la version originale de énoncés ait montré une fidélité, comme elle a été mesurée par l'alpha de Cronbach (1951), satisfaisante $(\alpha=0,81)$ (Holbrook, 1994), des analyses factorielles confirmatoires ont donné des résultats décevants pour un modèle unidimensionnel (Holbrook, 1993; Holbrook et Schindler, 1994). Cela a conduit à réduire l'échelle de 20 à 8 énoncés, dont la fidélité s'est révélée satisfaisante dans deux études ( $\alpha=0,78$ et 0,73 [Holbrook, 1993]) et à la limite de l'acceptable dans trois autres $(\alpha=0,68$ [Holbrook et Schindler, 1994; Zimmer, Little et Griffiths, 1999] et $\alpha=0,63$ [Rindfleisch, Freeman 
et Burroughs, 2000]). Cela a d'ailleurs incité l'auteur de l'échelle à retourner à la version originale de 20 énoncés, dont les propriétés psychométriques se sont révélées acceptables (Holbrook et Schindler, 1996; Schindler et Holbrook, 2003).

La faiblesse de la fidélité de la version à huit énoncés de l'index de nostalgie pourrait s'expliquer par la fatigue du répondant, due à la position de l'échelle à la fin d'un questionnaire long (Holbrook et Schindler, 1994), ou par le fait que certains énoncés sont à polarité inversée. En effet, l'incorporation d'énoncés à polarité inversée est susceptible de mettre en péril l'unidimensionnalité des échelles (Herche et Engelland, 1996). Cependant, la raison pourrait être liée au construit mesuré par cette échelle, qui serait intrinsèquement multidimensionnel. En effet, il est probable que les sentiments nostalgiques, ou selon nous la croyance au déclin, pour des produits soient distincts de ceux relatifs à la vie en général (Rindfleisch et Sprott, 2000). En particulier, les énoncés « Les changements technologiques garantiront un futur meilleur » et «Le monde des affaires construit constamment un avenir meilleur » ont dû être enlevés de l'échelle dans une étude internationale, car les opinions relatives à la technologie et au monde des affaires apparaissent comme n'étant pas reliées aux opinions envers la qualité de vie et envers la qualité des produits du passé (Steenkamp, Hofstede et Wedel, 1999).

Effectivement, deux études suggèrent que l'index de nostalgie (Holbrook, 1993, 1994) serait bidimension- nel (voir tableau 1). Spécifiquement, une analyse factorielle exploratoire sur les huit énoncés a révélé deux facteurs (Rindfleisch, Freeman et Burroughs, 2000). Un premier facteur, composé de trois énoncés, reflète une « nostalgie » spécifique aux produits $(\alpha=0,76)$ (p. ex. : « Les choses étaient mieux fabriquées dans le bon vieux temps ») et un second facteur, composé de quatre énoncés, reflète une "nostalgie » relative à la vie en général $(\alpha=0,75)$ (p. ex. : «L'Histoire implique une amélioration continue de la qualité de vie »; échelle inversée). Une autre analyse factorielle a révélé deux facteurs pour l'index de nostalgie à huit énoncés, un facteur « macro » $(\alpha=0,81)$, composé de quatre énoncés, nommé aussi propension à la nostalgie sociétale, et un facteur « micro » $(\alpha=0,78)$, composé de quatre énoncés, nommé aussi propension à la nostalgie individuelle (Reisenwitz, Iyer et Cutler, 2004). Ces deux analyses factorielles ont donné des résultats quasiment identiques : sept énoncés sur huit correspondent. L'énoncé qui a été exclu de l'analyse factorielle de Rindfleisch, Freeman et Burroughs (2000) se trouve être celui dont le "loading" est le moins élevé dans l'analyse factorielle de Reisenwitz, Iyer et Cutler (2004). En fait, ce résultat peut être interprété de la manière suivante. La question « We are experiencing a decline in the quality of life » est ambiguë en matière de niveau d'abstraction : il peut s'agir des conditions de vie matérielle immédiate (confort de la maison, de l'automobile, etc.), c'est-à-dire une qualité de vie « micro », ou des conditions de vie plus sociales (sentiment de sécurité, voisinage, etc.), c’est-à-dire une qualité de vie « macro».

\begin{tabular}{|c|c|c|}
\hline $\begin{array}{l}\text { Index de nostalgie - version à huit énoncés } \\
\text { (Holbrook et Schindler, 1994) }\end{array}$ & $\begin{array}{l}\text { Facteurs établis par } \\
\text { Rindfleisch, Freeman et } \\
\text { Burroughs, } 2000\end{array}$ & $\begin{array}{l}\text { Facteurs établis par } \\
\text { Reisenwitz, Iyer et } \\
\text { Cutler, 2004 }\end{array}$ \\
\hline 1 They don't make 'em like they used to & «Product nostalgia» & « Micro factor» \\
\hline $\begin{array}{l}2 \text { Things used to be better in the good old } \\
\text { days }\end{array}$ & «Product nostalgia » & « Micro factor » \\
\hline $\begin{array}{l}3 \text { Products are getting shoddier and } \\
\text { shoddier }\end{array}$ & «Product nostalgia » & «Micro factor» \\
\hline $\begin{array}{l}4 \text { Technological change will ensure a } \\
\text { brighter future (reverse coded) }\end{array}$ & «Life nostalgia » & «Macro factor» \\
\hline $\begin{array}{l}5 \text { History involves a steady improvement in } \\
\text { human welfare (reverse coded) }\end{array}$ & « Life nostalgia » & «Macro factor» \\
\hline $\begin{array}{l}6 \text { We are experiencing a decline in the } \\
\text { quality of life }\end{array}$ & $\begin{array}{l}\text { Énoncé exclu de la solution } \\
\text { à deux facteurs }\end{array}$ & «Micro factor» \\
\hline $\begin{array}{l}7 \text { Steady growth in GNP has brought } \\
\text { increased human happiness (reverse coded) }\end{array}$ & « Life nostalgia » & « Macro factor» \\
\hline $\begin{array}{l}8 \text { Modern business constantly builds a better } \\
\text { tomorrow (reverse coded) }\end{array}$ & « Life nostalgia » & « Macro factor» \\
\hline
\end{tabular}

Note : L'index de nostalgie de Holbrook comportait à l’origine 20 énoncés (Holbrook, 1993, 1994) et a ensuite été réduit à 8 énoncés (Holbrook et Schindler, 1994).

Tableau 1 - L'index de nostalgie de Holbrook : un indicateur multidimensionnel 
Cependant, l'explication principale de la fidélité problématique de l'échelle de nostalgie, et de sa multidimensionnalité, pourrait être d'ordre conceptuel. En effet, l'index de nostalgie (Holbrook, 1993, 1994) a été développé sur la base de la conceptualisation de la nostalgie de Davis (1979), qui considère la nostalgie comme " une évocation à connotation positive d'un passé vécu dans un contexte de sentiments négatifs envers le présent » (Davis, 1979, p. 18). Ainsi, l'index de nostalgie mesure des croyances selon lesquelles le passage du temps est associé à un déclin (Schindler et Holbrook, 2003), ce qui implique une comparaison entre le passé, le présent et le futur, où le passé est perçu comme supérieur. Dans cette vision de la nostalgie, l'attrait du passé est relié à des sentiments négatifs ou " dédain » envers le présent (Davis, 1979), et une inquiétude relative au futur (Davis, 1979; Nawas et Platt, 1965). Or, cette échelle mesurerait plutôt la croyance au déclin (Hallegatte et Marticotte, 2014), ce qui permettrait d'interpréter les dimensions comme l'une étant la croyance au déclin général et l'autre la croyance au déclin de la qualité des produits. Le premier serait du pessimisme, le deuxième de la clairvoyance sur le phénomène d'obsolescence programmée.

Compte tenu de ce qui précède, nous posons les hypothèses suivantes qui, si elles étaient soutenues, offriraient un soutien supplémentaire indirect à l'hypothèse $\mathrm{H}_{2}$ :

$\mathrm{H}_{3 \mathrm{a}}$ : La croyance au déclin comporte deux dimensions : une croyance au déclin global et une croyance au déclin spécifique.

$\mathrm{H}_{3 \mathrm{~b}}$ : La propension à la nostalgie est unidimensionnelle.

\section{L'âge de la nostalgie}

Un dernier élément, qui ne concerne pas la nature de la nostalgie, mais ses propriétés, reste encore obscur et mérite toute notre attention. Il s'agit de son lien avec l'âge. La croyance populaire voudrait que la propension à la nostalgie augmente avec l'âge. En effet, lorsqu'on avance en âge, le réservoir d'expériences passées augmente, en particulier le nombre de personnes rencontrées, d'événements vécus et de lieux fréquentés, et donc les objets potentiels de nostalgie deviennent plus nombreux. Reflétant cette croyance, Davis (1979) pensait que la nostalgie était plus fréquente lors du « dernier tiers » de la vie, quoique des pics de nostalgie fussent possibles lors de changements importants dans la vie (fin des études et entrée dans la vie active, passage au statut de parent, etc.).

Cependant, parmi les recherches empiriques sur le lien entre la nostalgie et l'âge, il n'y a pas d'étude, à notre connaissance, qui soutienne l'hypothèse selon laquelle la nostalgie augmente avec l'âge. Une étude montre que l'âge n'a pas d'influence significative sur la propension à la nostalgie, comme elle a été mesurée par l'inventaire nostalgique de Batcho (1995) (Merchant, Ford et Rose, 2011). Dans une autre étude, le lien entre la nostalgie et l'âge est statistiquement significatif, mais il est contradictoire avec l'idée selon laquelle la nostalgie augmente avec l'âge. Batcho (1995) trouve des différences statistiquement significatives de la propension à la nostalgie entre les âges, mais le pic de nostalgie se situerait aux alentours de la fin de l'adolescence et du début de la vingtaine. Cependant, la relation entre l'âge et la nostalgie dépendrait de l'objet de la nostalgie. Par exemple, la nostalgie pour la musique augmenterait de manière statistiquement significative avec l'âge, contrairement à la tendance globale (Batcho, 1995). Il est donc difficile de conclure globalement à l'existence ou l'absence de lien, d'autant plus que l'inventaire nostalgique de Batcho (1995), qui permet de déterminer à quel point certains éléments du passé manquent aux répondants, ne mesure qu'un aspect de la nostalgie qui est apparue par la suite comme une émotion complexe (Wildschut et al., 2006).

Par ailleurs, en ce qui concerne la croyance au déclin, plusieurs études montrent que l'index de nostalgie de Holbrook $(1993,1994)$ n'est pas corrélé de manière statistiquement significative avec l'âge (Holbrook, 1993, 1994; Holbrook et Schindler, 1994; Schindler et Holbrook, 2003), ce qui implique que la croyance au déclin, que mesure en fait l'index de nostalgie de Holbrook (Hallegatte et Marticotte, 2014; Schindler et Holbrook, 2003), n'est pas corrélée avec l'âge. En outre, une échelle issue du domaine de la psychologie environnementale, l'échelle "Antiquarianism Scale », issue de l'inventaire de réponse environnementale de McKechnie $(1974,1977)$, qui mesure un attrait pour les choses du passé, n’est pas corrélée de manière statistiquement significative avec l'âge du répondant (Schindler et Holbrook, 2003). En revanche, dans 
cette même étude de Schindler et Holbrook (2003), une autre échelle que celle citée précédemment, et issue de la psychologie environnementale, l'«échelle d'expérience», provenant d'une étude sur les dispositions personnelles envers le passé (Taylor et Konrad, 1980) et qui mesure une forme d'attrait envers les choses du passé, a été utilisée. Cet indicateur est effectivement corrélé avec l'âge, mais négativement, ce qui indiquerait que les plus jeunes sont plus attirés par le passé.

L'ensemble de ces résultats relatifs à la nostalgie et des concepts connexes suggère que l'âge soit n'a rien à voir, soit a un effet contraire à la croyance populaire. Nous sommes probablement en présence d'un phénomène complexe ou le lien entre l'âge et la nostalgie dépendrait du contexte ou de l'objet de la nostalgie. Mais, globalement, il n'y a pas de raisons de penser que la nostalgie augmente avec l'âge, que l'on peut être nostalgique très jeune, et que ceux qui partent étudier dans un endroit autre que leur lieu d'enfance pourraient vivre un pic de nostalgie dans leur vingtaine. Dit autrement, il n'y a pas de raison de penser qu'il existe une relation linéaire entre les deux variables.

Compte tenu du de qui précède nous posons l'hypothèse suivante :

$\mathrm{H}_{4}$ : La propension à la nostalgie est indépendante de l'âge de l'individu.

\section{Méthodologie}

Nous avons choisi la musique populaire comme contexte pour tester nos hypothèses, d'abord parce que la caractéristique principale de ce secteur depuis le début du siècle est qu'il est résolument tourné vers le passé (Reynolds, 2011), et que la longévité de certaines marques « artistiquement inactives » comme Elvis, The Beatles, Bob Marley ou Frank Sinatra est pour le moins surprenante pour tout spécialiste du marketing, étant donné le changement rapide des goûts, des caprices des consommateurs, de l'intense concurrence et du rythme auquel sortent de nouveaux produits dans l'industrie de la musique (Blackwell et Stephan, 2004). Mais surtout, la musique apparait comme un produit artistique de consommation de masse susceptible de susciter des sentiments nostalgiques pour l'ensemble des consommateurs. Dans le domaine de la musique, la consommation passée est particulièrement importante (Shankar, Elliott et Fitchett, 2009) et les goûts musicaux développés à la fin de l'adolescence et le début de l'âge adulte influencent de façon durable les préférences des individus dans la suite de leur vie (Holbrook et Schindler, 1989). D'ailleurs, la nostalgie pour l'adolescence et le début de l'âge adulte serait plus forte que pour n'importe quelle autre période (Davis, 1979). Il n'est donc pas étonnant que la musique populaire, objet esthétique de l'adolescence par excellence, soit un thème récurrent de l'expérience nostalgique (Holak et Havlena, 1992) et qu'elle constitue le type de "produit nostalgique » choisi par le plus grand nombre de répondants $(33 \%)$ dans l'étude de Sierra et McQuitty (2007). Plusieurs autres études en psychologie suggèrent aussi que la musique suscite la nostalgie (Barrett et al., 2010; Janataa, Tomic et Rakowskia, 2007; Juslin et al., 2008; Zentner, Grandjean et Scherer, 2008). La musique joue aussi un rôle central dans la production de nostalgie dans les « films nostalgiques » (Shumway, 1999), peutêtre parce qu'elle représente des styles de vie utopiques, tels que l'« hédonisme californien » symbolisé par le groupe de musique The Beach Boys (Askegaard, 2010).

Afin de choisir un ou plusieurs groupes de musique les plus pertinents pour nos investigations, nous avons examiné les six caractéristiques de Brown, Kozinets et Sherry Jr. (2003a, 2003b) qu'une marque devrait posséder afin de pouvoir faire l'objet de rétromarquage (retrobranding), stratégie qui consiste à conserver l'association d'une marque au passé (Hallegatte, 2014), courante voire systématique dans la musique populaire (Reynolds, 2011). Au moins deux groupes de musique populaire, ayant obtenu un succès immense dans les années 1970, apparaissent répondre en tous points à ces critères : il s'agit de Led Zeppelin, et ABBA. En outre, Led Zeppelin a été l'objet d'un buгz médiatique autour d'un concert unique à Londres le 10 décembre 2007 et d'une possible reformation. En ce qui concerne ABBA, selon un sondage effectué en Grande-Bretagne en 2009, il serait aussi le groupe que le plus de personnes voudraient voir reformer, loin devant les deuxième et troisième groupes de ce palmarès, The Police et Pink Floyd ${ }^{2}$. La sortie du film "Mamma Mia! », basé sur les chansons du groupe ABBA, en 2008, n'y est certainement pas étrangère. 
Nous avons donc effectué deux études. Pour la première, la population à l'étude était l'ensemble des personnes susceptibles d'assister à un concert de Led Zeppelin, que nous avons atteinte en postant des billets d'invitation sur des forums de discussion spécialisés dans Internet. Le questionnaire a été programmé sur un logiciel de collecte de données en ligne (SurveyMonkey), et prétesté du 18 au 24 janvier 2012 auprès de 28 personnes. La collecte de données principale a pris fin le 20 février 2012, avec 181 questionnaires exploitables. Pour la deuxième, la population à l'étude était composée des amateurs du groupe ABBA que nous avons atteinte en postant des billets d'invitation sur des forums de discussion spécialisés dans Internet, mais aussi via ABBA Intermezzo, un véritable fanclub institué, basé en Allemagne, qui publie une revue papier consacrée à $\mathrm{ABBA}$, plusieurs fois par année. Du 15 mars au 26 avril 2012, nous avons obtenu 312 questionnaires exploitables. Comme Led Zeppelin et ABBA sont deux groupes au style musical, à l'imagerie et aux connotations très différentes, il est raisonnable de penser qu'il s'agisse de deux populations distinctes.

La propension à la nostalgie a été mesurée par la Southampton Nostalgia Scale (SNS) (Routledge et al., 2008) parce qu'elle constitue une mesure unidimensionnelle, parcimonieuse et directe de la propension à la nostalgie. La SNS possède une consistance interne satisfaisante, comme elle a été mesurée par l'alpha de Cronbach, dont la valeur varie de 0,84 à 0,93 dans cinq études (Barrett et al., 2010; Juhl et al., 2010; Routledge et al., 2008). En outre, nous prévoyions une collecte internationale avec un questionnaire en anglais et un échantillon majoritairement nord-américain. Notons que cette échelle comporte le mot "nostalgie » dans ses énoncés, mais inclure une définition apparaît inutile (Wildschut et al., 2006; Wildschut et al., 2010). En effet, les " théories naïves " (lay conceptions) qu'ont les gens à propos de ce qu'est la nostalgie correspondent assez bien à la façon dont la nostalgie est considérée depuis plusieurs années en psychologie (Hepper et al., 2012) et en marketing. De plus, l'inclusion ou l'absence d'inclusion d'une définition formelle de la nostalgie dans le questionnaire n'a pas eu d'effet sur les résultats des deux études (Wildschut et al., 2006; Wildschut et al., 2010). L'index de nostalgie de Holbrook (1993, 1994) n'est pas apparu approprié, car il s'agit d'une mesure indirecte (Schindler et Holbrook, 2003) si ce n'est invalide (Hallegatte et Marticotte, 2014) de la propension à la nostalgie.

La croyance au déclin a été mesurée par l'index de nostalgie de Holbrook (1993, 1994), dans sa version à huit énoncés (Holbrook et Schindler, 1994), puisque l'auteur même des différentes versions de cette échelle considère qu'elle mesure la croyance selon laquelle le passage du temps est associé à un déclin (Schindler et Holbrook, 2003) et que cette croyance est distincte de la propension à la nostalgie (Hallegatte et Marticotte, 2014). De plus, celle-ci comporte deux dimensions (Reisenwitz, Iyer et Cutler, 2004; Rindfleisch, Freeman et Burroughs, 2000), soit une croyance au déclin globale, et une croyance au déclin spécifique (de la qualité des produits), que nous avons adaptées à notre contexte, la musique populaire, et que nous appellerons donc croyance au déclin musical.

Pour mesurer le passé positif, nous utiliserons un sous-ensemble de huit énoncés du Zimbardo Time Perspective Inventory (ZTPI) (Zimbardo et Boyd, 1999) utilisé par Routledge et al. (2008) comme mesure d'attitude envers le passé, mais nommé «passé positif » par son auteur. Cette variable a été incluse dans la deuxième étude (ABBA) afin d'enrichir l'analyse. Par ailleurs, pour mesurer l'attachement à la marque, nous avons utilisé l'échelle de Park et al. (2010), qui inclut la connexion de la marque avec le soi (Escalas et Bettman, 2005; Sprott, Czellar et Spangenberg, 2009) ainsi que son accessibilité cognitive (Park et al., 2010).

Pour la première étude (Led Zeppelin), les répondants étaient originaires principalement du Canada (38,2 \%), des États-Unis $(28,9 \%)$ et de la France $(20,4 \%)$. La classe d'âge la plus nombreuse était celle des 45 à 54 ans (31,6\%), mais toutes les classes d'âge étaient représentées. Comme l'échantillon était composé très majoritairement d'hom-mes (82,2\%), nous avons vérifié que le sexe n'était pas lié à la propension à la nostalgie, ce qui était le cas $\left(t_{150}=0,629 ; p=0,530\right)$. Pour la deuxième étude (ABBA), l'échantillon était composé à $61,5 \%$ d'hommes et 38,5\% de femmes, soit au-delà de deux fois plus de femmes que celui de la première étude $(17,8 \%)$. En ce qui concerne l'âge, la moitié de l'échantillon $(50,4 \%)$ avait de 45 à 54 ans. Les jeunes de 15 à 24 ans $(6,9 \%)$ et 
de 25 à 34 ans (9,4\%), qui n’ont pu connaître la période d'activité artistique du groupe, étaient nettement moins nombreux qu'à la première étude (respectivement $21,7 \%$ et 18,4\%). Ainsi, l'échantillon de cette deuxième étude était composé de personnes nettement plus âgées que celui de la première étude. Par ailleurs, les répondants résidaient dans 37 pays différents : $23 \%$ en Allemagne, 16,6\% au Royaume-Uni, 7,7 \% aux ÉtatsUnis et $5,1 \%$ en Australie. Tous les autres pays, en très grande majorité européens, comptaient pour moins de $5 \%$ de l'échantillon chacun.

\section{Résultats}

Avant de tester nos hypothèses, nous avons effectué les vérifications préliminaires d'usage, soit la fidélité des mesures des construits à indicateur multiple, comme elle a été mesurée par l'indicateur de consistance interne alpha de Cronbach, qui varie de satisfaisante (alpha $>0,75)$ à excellente (alpha $>0,9)$. Ensuite, nous avons croisé les variables à l'étude, calculé le coefficient de corrélation et effectué un test statistique de significativité pour chacune des deux études (voir tableaux 2 et 3 ).

\begin{tabular}{|c|c|c|c|c|c|c|}
\hline & & $\begin{array}{l}\text { Propension à } \\
\text { la nostalgie }\end{array}$ & $\begin{array}{l}\text { Croyance au } \\
\text { déclin musical }\end{array}$ & $\begin{array}{l}\text { Croyance au } \\
\text { déclin global }\end{array}$ & Attachement & Âge \\
\hline $\begin{array}{l}\text { Propension à la } \\
\text { nostalgie }\end{array}$ & $\begin{array}{l}\mathrm{r} \\
p\end{array}$ & 1 & $\begin{array}{l}0,297^{* *} \\
0,000\end{array}$ & $\begin{array}{l}-0,076 \\
0,345 \\
\end{array}$ & $\begin{array}{l}-0,030 \\
0,710\end{array}$ & $\begin{array}{l}0,031 \\
0,706 \\
\end{array}$ \\
\hline $\begin{array}{l}\text { Croyance au } \\
\text { déclin musical }\end{array}$ & $\begin{array}{l}\mathrm{r} \\
p\end{array}$ & $\begin{array}{l}0,297^{* *} \\
0,000\end{array}$ & 1 & $\begin{array}{l}0,118 \\
0,141 \\
\end{array}$ & $\begin{array}{l}0,109 \\
0,176 \\
\end{array}$ & $\begin{array}{l}0,018 \\
0,826 \\
\end{array}$ \\
\hline $\begin{array}{l}\text { Croyance au } \\
\text { déclin global }\end{array}$ & $\mathrm{r}$ & $\begin{array}{l}-0,076 \\
0,345 \\
\end{array}$ & $\begin{array}{l}0,118 \\
0,141 \\
\end{array}$ & 1 & $\begin{array}{l}0,264^{* *} \\
0,001\end{array}$ & $\begin{array}{l}-0,093 \\
0,255\end{array}$ \\
\hline $\begin{array}{l}\text { Attachement à } \\
\text { la marque }\end{array}$ & $\mathrm{r}$ & $\begin{array}{l}-0,030 \\
0,710 \\
\end{array}$ & $\begin{array}{l}0,109 \\
0,176\end{array}$ & $\begin{array}{l}0,264^{* *} \\
0,001 \\
\end{array}$ & 1 & $\begin{array}{l}0,012 \\
0,882\end{array}$ \\
\hline Âge & $\begin{array}{l}\mathrm{r} \\
p\end{array}$ & $\begin{array}{l}0,031 \\
0,706 \\
\end{array}$ & $\begin{array}{l}0,018 \\
0,826\end{array}$ & $\begin{array}{l}-0,093 \\
0,255 \\
\end{array}$ & $\begin{array}{l}0,012 \\
0,882\end{array}$ & 1 \\
\hline
\end{tabular}

** La corrélation est significative au niveau 0,01 (test bilatéral)

Tableau 2 - Corrélations bivariées de la première étude (Led Zeppelin) -

\begin{tabular}{|c|c|c|c|c|c|c|c|}
\hline & & $\begin{array}{l}\text { Propension } \\
\text { à la } \\
\text { nostalgie }\end{array}$ & $\begin{array}{l}\text { Croyance } \\
\text { au déclin } \\
\text { musical }\end{array}$ & $\begin{array}{l}\text { Croyance } \\
\text { au déclin } \\
\text { global }\end{array}$ & Attachement & Passé positif & $\hat{A} g e$ \\
\hline $\begin{array}{l}\text { Propension à } \\
\text { la nostalgie }\end{array}$ & $\begin{array}{l}\mathrm{r} \\
p\end{array}$ & 1 & $\begin{array}{l}0,355^{* *} \\
0,000\end{array}$ & $\begin{array}{l}0,041 \\
0,493\end{array}$ & $\begin{array}{l}0,239^{* *} \\
0,000\end{array}$ & $\begin{array}{l}0,538^{* *} \\
0,000\end{array}$ & $\begin{array}{l}-0,001 \\
0,982\end{array}$ \\
\hline $\begin{array}{l}\text { Croyance au } \\
\text { déclin musical }\end{array}$ & $\begin{array}{l}\mathrm{r} \\
p\end{array}$ & $\begin{array}{l}0,355^{* *} \\
0,000\end{array}$ & 1 & $\begin{array}{l}0,177^{* *} \\
0,002\end{array}$ & $\begin{array}{l}0,225^{* *} \\
0,000\end{array}$ & $\begin{array}{l}0,260^{* *} \\
0,000\end{array}$ & $\begin{array}{l}-0,075 \\
0,215\end{array}$ \\
\hline $\begin{array}{l}\text { Croyance au } \\
\text { déclin global }\end{array}$ & $\begin{array}{l}\mathrm{r} \\
p\end{array}$ & $\begin{array}{l}0,041 \\
0,493\end{array}$ & $\begin{array}{l}0,177^{* *} \\
0,002\end{array}$ & 1 & $\begin{array}{l}0,215^{* *} \\
0,000\end{array}$ & $\begin{array}{l}0,115 \\
0,057\end{array}$ & $\begin{array}{l}0,008 \\
0,899\end{array}$ \\
\hline $\begin{array}{l}\text { Attachement } \\
\text { à la marque }\end{array}$ & $\begin{array}{l}\mathrm{r} \\
p\end{array}$ & $\begin{array}{l}0,239^{* *} \\
0,000\end{array}$ & $\begin{array}{l}0,225^{* *} \\
0,000\end{array}$ & $\begin{array}{l}0,215^{* *} \\
0,000\end{array}$ & 1 & $\begin{array}{l}0,217^{* *} \\
0,000\end{array}$ & $\begin{array}{l}-0,047 \\
0,437\end{array}$ \\
\hline Passé positif & $\begin{array}{l}\mathrm{r} \\
p\end{array}$ & $\begin{array}{l}0,538^{* *} \\
0,000\end{array}$ & $\begin{array}{l}0,260^{* *} \\
0,000\end{array}$ & $\begin{array}{l}0,115 \\
0,057\end{array}$ & $\begin{array}{l}0,217^{* *} \\
0,000\end{array}$ & 1 & $\begin{array}{l}0,062 \\
0,311\end{array}$ \\
\hline$\hat{A} g e$ & $\begin{array}{l}\mathrm{r} \\
p\end{array}$ & $\begin{array}{l}-0,001 \\
0,982\end{array}$ & $\begin{array}{l}-0,075 \\
0,215\end{array}$ & $\begin{array}{l}0,008 \\
0,899\end{array}$ & $\begin{array}{l}-0,047 \\
0,437\end{array}$ & $\begin{array}{l}0,062 \\
0,311\end{array}$ & 1 \\
\hline
\end{tabular}

** La corrélation est significative au niveau 0,01 (test bilatéral)

* La corrélation est significative au niveau 0,05 (test bilatéral) 
Le passé positif, variable incluse dans la deuxième étude seulement, est assez fortement corrélé avec la propension à la nostalgie $(r=0,538 ; p<0,001)$. Ainsi, l'hypothèse $H_{1}$, selon laquelle « la nostalgie est reliée à un passé positif » est soutenue par nos données. Il est à noter que le passé positif est aussi corrélé, mais faiblement, avec la croyance au déclin musical $(r=0,260 ; p=0,000)$, mais pas significativement avec la croyance au déclin global $(r=0,115$; $p=0,057)$.

Dans la première étude, la propension à la nostalgie est faiblement corrélée avec la croyance au déclin musical $(r=0,297 ; p<0,000)$, et pas du tout avec la croyance au déclin global $(r=-0,076$; $p=0,345)$. Les résultats sont quasiment identiques dans la deuxième étude (respectivement $r=0,355$; $p<0,001 ; r=0,041 ; p=0,493)$. Ainsi, l'hypothèse $\mathrm{H}_{2}$, selon laquelle « la propension à la nostalgie et la croyance au déclin sont des concepts distincts» est soutenue.

Quant aux deux dimensions de la croyance au déclin, elles ne sont pas significativement corrélées entre elles dans la première étude $(r=0,118$; $p=0,141)$ et très faiblement corrélées dans la deuxième $(r=0,177 ; p=0,002)$. Par la suite, nous avons effectué des analyses en composantes principales pour la croyance au déclin, qui a produit une solution à deux dimensions dans les deux études (respectivement $65,6 \%$ et $69,8 \%$ de variance expliquée), très facilement interprétable, surtout après la rotation orthogonale qui a donné la solution la plus claire, l'approche Quartimax avec normalisation de Kaiser. Cette solution est conforme à ce qui était attendu : les trois premiers énoncés mesurent une croyance au déclin spécifique à la musique et les quatre suivants, une croyance au déclin global. Nous avons ensuite vérifié la consistance interne (alpha de Cronbach) de la mesure de chacune de ces deux dimensions : les scores sont acceptables, soit 0,77 pour la croyance au déclin musical et 0,80 pour la croyance au déclin global. Ainsi, l'hypothèse $\mathrm{H}_{3 a}$, selon laquelle " la croyance au déclin comporte deux dimensions : une croyance au déclin global et une croyance au déclin spécifique » est soutenue.

Nous avons vérifié l'unidimensionnalité de la propension à la nostalgie par une analyse en composante principale. Les résultats ont produit une solution à un facteur dans les deux études, le premier facteur extrait expliquant respectivement $83 \%$ et $76,2 \%$ de la variance. De plus, l'alpha de Cronbach correspondant s'est montré très élevé, respectivement de 0,95 et 0,92 , malgré la présence d'un énoncé à polarité inversée, susceptible d'affecter négativement l'unidimensionnalité de l'échelle (Herche et Engelland, 1996). Ainsi, l'hypothèse $\mathrm{H}_{3 \mathrm{~b}}$, selon laquelle « la propension à la nostalgie est unidimensionnelle » est soutenue.

En outre, l'âge n'est corrélé avec aucune des autres variables à l'étude dans les deux études : ni avec la propension à la nostalgie (respectivement $r=0,031 ; p=0,706 ; r=-0,001 ; p=0,982)$, ni avec la croyance au déclin musical $(r=0,018 ; p=0,826$; $r=-0,075 ; p=0,215)$, ni avec la croyance au déclin global $(r=-0,093 ; p=0,255 ; r=0,008 ; p=0,899)$, ni avec l'attachement $(r=0,012 ; p=0,882 ; r=$ $-0,047 ; p=0,437)$, ni avec le passé positif $(r=0,062$; $p=0,311)$. Ainsi, l'hypothèse $\mathrm{H}_{4}$, selon laquelle « la propension à la nostalgie ne dépend pas de l'âge " est soutenue.

\section{Discussion}

Nos résultats soutiennent empiriquement les arguments théoriques de Hallegatte et Marticotte (2014) selon lesquels la propension à la nostalgie est un concept distinct de la croyance au déclin, contrairement à la position de Holbrook et Schindler (1994) selon laquelle il s'agit de synonymes, et de celle de Schindler et Holbrook (2003) selon laquelle le deuxième est un indicateur du premier. En effet, nos résultats ont montré que la croyance au déclin est très peu corrélée avec la propension à la nostalgie dans nos deux études. Spécifiquement, la croyance au déclin musical est faiblement corrélée avec la propension à la nostalgie alors que la croyance au déclin global ne l'est pas du tout. Les conséquences de ces résultats sont importantes puisque l'index de nostalgie de Holbrook (1993, 1994), censé initialement mesurer la propension à la nostalgie (ou encore l'attitude envers le passé), a été requalifié ultérieurement par Holbrook luimême comme mesure de la croyance au déclin (Schindler et Holbrook, 2003). En considérant a posteriori que « la croyance que le passage du temps est associé à un déclin » est une mesure de la propension à la nostalgie (Schindler et Holbrook, 2003, p. 297), l'auteur de l'index de nostalgie a éludé habilement le problème de validité de son échelle 
que nous avons soulevé, mais a rajouté à la confusion. Néanmoins, puisque l'index de nostalgie de Holbrook mesure la croyance au déclin, et que la croyance au déclin est distincte de la propension à la nostalgie, l'index de nostalgie de Holbrook $(1993,1994)$ est invalide.

Pour comprendre cela, il faut rappeler que l'élaboration de l'index de nostalgie de Holbrook s'appuie explicitement sur la conceptualisation de la nostalgie de Davis (1979), aujourd'hui partiellement dépassée. En effet, les avancées des connaissances ont remis en question la conceptualisation de Davis, et notamment l'idée que la nostalgie, ou une vision positive du passé, n'est pas opposée à une satisfaction envers le présent, ou à une orientation vers le futur (Batcho, 1995, 1998; Godbole, Shehryar et Hunt, 2006; Spears et Amos, 2012; Zimbardo et Boyd, 1999). Dit autrement, la croyance au déclin, qui, par définition, met en opposition le passé avec le présent et le futur, ne peut pas être considérée comme l'équivalent de l'attrait pour le passé et encore moins de la propension à la nostalgie. Au moment de l'élaboration de l'échelle, les recherches en marketing sur le sujet en étaient à leurs balbutiements, la vision pessimiste de la nostalgie prévalait, et son invalidité pouvait donc difficilement être établie. En revanche, il est troublant que, 10 ans plus tard, Schindler et Holbrook (2003) n'ont pas reconnu l'invalidité de l'index de nostalgie de Holbrook et, plus largement, la distinction conceptuelle opérée par Hallegatte et Marticotte (2014) et soutenue par les données de la présente étude.

Ces résultats imposent, au mieux, de requalifier et, au pire, d'invalider l'ensemble des recherches dans la littérature scientifique utilisant cet instrument de mesure. Les chercheurs en marketing, qui se fiaient à l'index de nostalgie de Holbrook (1993, 1994), désavoué à demi-mot par Holbrook lui-même (Schindler et Holbrook, 2003), pour mesurer la propension à la nostalgie, mesuraient la croyance au déclin sans le savoir. Contrairement à M. Jourdain qui faisait de la prose sans le savoir, dans Le Bourgeois gentilhomme de Molière, ces chercheurs ont une part de responsabilité, peut-être celle d'avoir été aveuglés par une grande marque, la marque «Holbrook ». Morris Holbrook est probablement le Paul McCartney du marketing, et son statut de démiurge incite probablement à penser que ses productions sont généralement de haute qualité, ce qui ne fait aucun doute, mais au moins une exception existe, et ce sont ses travaux sur la nostalgie. On peut penser que la communauté scientifique n'a pas été assez critique à l'égard de ses travaux, alors que, contrairement à la production de Paul McCartney, celle de Holbrook concernant la nostalgie a mal vieilli. L'index de nostalgie souffre de problèmes de validité depuis sa conception, qui se sont aggravés au fil du temps en raison de l'évolution des connaissances, mais qui n'ont pas encore été largement admis par la communauté scientifique en marketing. Cependant, il n'y a pas lieu d'être nostalgique de l'index de nostalgie de Holbrook, puisque celuici a sa raison d'être en marketing : il mesure, de manière apparemment valide et fidèle, la croyance au déclin.

Comme évoqué précédemment, on pourrait considérer que les conséquences négatives directes de cet imbroglio conceptuel sont peu importantes : il suffirait de relire ces recherches en remplaçant "propension à la nostalgie » (ou " attitude envers le passé » utilisé fréquemment comme synonyme, ce qui nous apparaît comme révélateur d'une confusion conceptuelle) par " croyance au déclin ». Cependant, le marketing, comme discipline, a peutêtre perdu 25 ans d'efforts pour développer un instrument qui mesure réellement la propension à la nostalgie. En effet, dès le départ, la définition de la nostalgie de Holbrook et Schindler (1991) était très différente de celle de Belk (1990). L'ensemble des définitions proposées par la suite, en marketing ou dans d'autres disciplines, a marginalisé toujours davantage celle de Holbrook et Schindler (1991). Cela aurait dû mettre la puce à l'oreille à des chercheurs en marketing. Mais, au lieu de cela, l'index de nostalgie de Holbrook (1993, 1994) a été, de loin, la mesure de la propension à la nostalgie la plus utilisée en marketing. Fort heureusement, l'immense majorité des recherches ont porté sur la nostalgie ressentie, et ne sont donc pas touchées par le problème. Il n'y a donc pas de problème de validité de la recherche sur la nostalgie prise dans son ensemble. Toujours est-il qu'il nous apparait raisonnable de penser, si l'on tient compte du fait que la croyance au déclin, mesurée par l'index de nostalgie de Holbrook, n'a eu aucun effet significatif dans nos deux études, que d'autres études utilisant cette mesure invalide de la propension à la nostalgie ont été réalisées mais non publiées, faute d'effets significatifs. Si c'est le cas, l'avancée des connaissances sur la nostalgie en marketing a été 
sérieusement ralentie par ce problème de validité que nous avons mis en évidence.

En outre, nos résultats soutiennent que la croyance au déclin est un construit bidimensionnel, autant par l'analyse des corrélations que par les analyses en composantes principales, c'est-à-dire qu'il existe une croyance au déclin général des conditions de vie et une croyance au déclin spécifique, de la musique populaire dans le cas de notre étude. Nos résultats font écho à ceux de Rindfleisch, Freeman et Burroughs (2000) et Reisenwitz, Iyer et Cutler (2004), mais nous les avons interprétés à l'aune du concept de croyance au déclin. Cette interprétation nouvelle est importante puisqu'elle montre que l'on peut croire que la musique, c'était mieux avant, mais que cela n'implique pas de croire que la vie était mieux avant.

Cette bidimensionnalité de la croyance au déclin jette un discrédit supplémentaire sur les études ayant considéré l'index de nostalgie de Holbrook comme unidimensionnel. Mais surtout, sachant que nos données soutiennent l'unidimensionnalité de la propension à la nostalgie, ils soutiennent indirectement que la propension à la nostalgie et la croyance au déclin sont des construits distincts, contrairement à la vision de Holbrook et Schindler (1994) selon laquelle il s'agit de synonymes et celle de Schindler et Holbrook (2003) selon laquelle le deuxième est un indicateur du premier. En effet, outre le manque de corrélation entre les deux échelles, la bidimensionnalité de l'échelle de croyance au déclin impliquerait, si elle mesurait la propension à la nostalgie, que cette dernière serait bidimensionnelle, et commanderait une explication théorique, inexistante dans la littérature à notre connaissance. Par conséquent, la bidimensionnalité de la croyance au déclin et l'unidimensionnalité de la propension à la nostalgie corroborent l'idée que l'index de nostalgie de Holbrook (1993, 1994), qui mesure la croyance selon laquelle le passage du temps est associé à un déclin (Schindler et Holbrook, 2003), est une mesure invalide de la propension à la nostalgie.

Par ailleurs, nous avons soutenu qu'ancien et nostalgique ne sont pas synonymes, et que seul un individu peut être nostalgique. Ce laxisme sémantique est révélateur, selon nous, de confusions conceptuelles, et ne saurait être considéré comme anecdotique. Cela nous a amené à distinguer quatre concepts : ancienneté perçue de l'objet (produit, marque, publicité), nostalgie ressentie, propension à la nostalgie et réponse attitudinale ou comportementale (voir figure 1). La simplicité de cette distinction rend difficilement explicable l'imbroglio sémantico-conceptuel mis en évidence. Le fait que Holbrook lui-même ait introduit l'expression fautive de «préférence nostalgique » (Holbrook, 1993) n'y est certainement pas étranger. Ce péché originel a probablement ouvert la voie au dérapage sémantique des 25 dernières années, et celui-ci a certainement favorisé la confusion conceptuelle discutée précédemment. D'ailleurs, 10 années plus tard, le porte-étendard de la recherche sur la nostalgie en marketing naviguait encore en eaux troubles avec l'expression « attachement nostalgique » (Schindler et Holbrook, 2003). Cette dernière expression ne pourrait être qu'un pléonasme anodin, puisque la nostalgie reflète une forme d'attachement au passé, mais contribue malheureusement à la confusion ambiante.

Nous avons aussi soutenu l'idée que nostalgie n'est pas une préférence pour le passé, en conformité avec le quasi-consensus interdisciplinaire sur le sujet, que l'on pourrait qualifier de consensus sans la définition de Holbrook et Schindler (1991) maintes fois reprises dans la recherche en marketing jusqu'à aujourd'hui. En revanche, nos données ont soutenu empiriquement l'idée que la propension à la nostalgie est reliée à un passé positif. Dit autrement, être nostalgique serait le privilège de ceux qui ont eu une vie heureuse. Cette idée selon laquelle la nostalgie est reliée au passé positif n'est pas complètement nouvelle (Godbole, Shehryar et Hunt, 2006), mais notre étude a établi le premier lien empirique direct entre la propension à la nostalgie et le passé positif, à notre connaissance.

Finalement, nos résultats soutiennent que la propension à la nostalgie n'augmente pas avec l'âge. Cela est cohérent avec la littérature existante selon laquelle soit la propension à la nostalgie ne dépend pas de l'âge (Merchant, Ford et Rose, 2011), soit elle varie en fonction de l'âge mais de manière non linéaire, c'est-à-dire que le pic de propension à la nostalgie ne se situe pas en fin de vie (Batcho, 1995). Nos données soutiennent une absence de corrélation. Ajoutons, même si nous considérons que l'index de nostalgie de Holbrook est une mesure invalide de la propension à la nostalgie, qu'il est intéressant de noter que Holbrook soutient 
l'idée d'absence de corrélation entre l'âge et la propension à la nostalgie (Holbrook, 1993, 1994; Holbrook et Schindler, 1994; Schindler et Holbrook, 2003), voire de corrélation opposée, c'est-à-dire que plus on est jeune, plus on serait prompt à la nostalgie (Schindler et Holbrook, 2003). Dans tous les cas, aucune donnée empirique, à notre connaissance, ne soutient l'idée que la propension à la nostalgie augmente avec l'âge. Nos résultats devraient contribuer à faire disparaitre cette idée reçue selon laquelle la propension à la nostalgie augmente avec l'âge et suggèrent de ne pas considérer a priori l'âge comme variable de segmentation dans le rétromarketing. En effet, comme l'âge et la propension à la nostalgie ne sont pas reliés, l'âge ne peut malheureusement pas être utilisé comme variable de substitution (proxy).

Nos données n'ont pas non plus montré de lien significatif entre l'âge et la croyance au déclin, qu'il s'agisse de croyance au déclin musical ou de croyance au déclin global, ces deux dimensions étant clairement distinctes. Nos données sont cohérentes avec la littérature, et aussi probablement en contradiction avec la croyance populaire. En effet, l'index de nostalgie de Holbrook (1993, 1994), qui mesure en fait la croyance au déclin, n'était pas corrélé de manière statistiquement significative avec l'âge dans des études précédentes (Holbrook, 1993, 1994; Holbrook et Schindler, 1994; Schindler et Holbrook, 2003). Le passé positif, quant à lui, n'est pas non plus corrélé avec l'âge, en cohérence avec les résultats de Zimbardo et Boyd (1999). En fait, ni la dimension " passé positif », ni la dimension « passé négatif » de l'inventaire de perspective temporelle de Zimbardo (ZTPI) n'étaient corrélées avec l'âge, alors que la dimension « futur » l'était.

Ajoutons, de plus, que nos résultats montrent que l'attachement à la marque (c'est-à-dire au groupe de musique Led Zeppelin ou à ABBA) n'est pas non plus relié à l'âge. Bien que les consommateurs plus âgés restent attachés plus longtemps à une même marque que les plus jeunes (Lambert-Pandraud et Laurent, 2010), nos résultats suggèrent que l'intensité de l'attachement à un groupe de musique rétro n'est pas reliée à l'âge. Les gestionnaires de marketing ne devraient donc pas tenir pour acquis qu'un consommateur qui a vécu directement la période de référence (les années 1970 dans nos études) est plus attaché à la marque rétro (le groupe de musique) qu'un consommateur trop jeune pour cela.

Ainsi, ni la propension à la nostalgie, ni la croyance au déclin, ni le passé positif, ni l'attachement à la marque ne dépendent de l'âge. Ces résultats sont de nature à faire disparaître des idées reçues, mais aussi à comprendre pourquoi les produits rétro touchent manifestement tous les groupes d'âge, qui peuvent tous être attachés à un objet du passé, même s'il ne s'agit pas d'un passé vécu directement. Par contre, il est important de préciser que ces résultats ne sont pas en contradiction avec l'idée selon laquelle les jeunes sont plus orientés vers le futur, puisque l'orientation temporelle " passé positif » n'est pas corrélée avec l'orientation temporelle "présent » et « futur» (Zimbardo et Boyd, 1999) ou, plus globalement, une orientation vers le passé n'est pas opposée à une orientation temporelle vers le futur ou le présent (Spears et Amos, 2012).

Cependant, comme toute recherche empirique, les résultats obtenus dans cette recherche sont contingents au contexte choisi, à la manière de procéder, aux variables incluses, aux échelles de mesure choisies, aux échantillons utilisés, etc. Dans des domaines où la nostalgie est réelle mais probablement moins intense, comme l'automobile ou le sport, il est possible que les liens mis en évidences prennent une autre forme. Aussi, avec un échantillon composé de personnes moins fans de musique, mais qui pourraient tout autant ressentir de la nostalgie, il est possible, par exemple, que leur croyance au déclin soit reliée différemment la leur propension à la nostalgie. En outre, l'acceptabilité sociale de la nostalgie est susceptible de varier d'un pays à un autre, ce qui pourrait induire des résultats différents dans un contexte non-occidental. Vue l'évolution très rapide du rapport à la nostalgie dans les pays occidentaux, étendre l'investigation de la nostalgie à d'autres régions du monde apparait comme un axe de recherche prometteur. Plus globalement, les résultats obtenus sont limités par l'approche positiviste employée. La consommation de la musique populaire est une forme de consommation hédonique difficile à appréhender dans toute sa complexité en élaborant des construits et en investiguant sur leurs relations (Hirschman et Holbrook, 1982), tout comme la nostalgie elle-même. 


\section{Conclusion}

Cette recherche a soutenu empiriquement qu'être prompt à la nostalgie n'équivaut pas à croire au déclin des conditions de vie, que cette croyance n'a rien à voir avec la croyance au déclin spécifique de la musique, que l'on est plus prompt à la nostalgie si on a été heureux dans le passé et que tout cela n'a rien à voir avec l'âge. En outre, nous avons soutenu qu'être prompt à la nostalgie n'équivaut pas à préférer des choses du passé et que le fait qu'un objet soit perçu comme ancien n'implique pas automatiquement la nostalgie. Tous ces résultats permettent de conclure que la nostalgie du $21^{\mathrm{e}}$ siècle n'est vraiment plus ce qu'elle était.

\section{NOTES}

1 Traduction libre.

2 The Telegraph (2009, 25 novembre). Abba is the band most people want to see reform.

\section{RÉFÉRENCES}

Askegaard, S. (2010). Experience economy in the making: hedonism, play and coolhunting in automotive song lyrics. Consumption, Markets \& Culture, 13(4), 351-371.

Bambauer-Sachse, S. et Heribert, G. (2009). Effects of nostalgic advertising through emotions and the intensity of the evoked mental images ». Dans M. C. Campbell, J. J. Inman et R, Pieters (dir.), Advances in consumer research (vol. 36, p. 391-398). Duluth, MN: Association for Consumer Research.

Barrett, F. S., Grimm, K. J., Robins, R. W., Wildschut, T., Sedikides, C. et Janata, P. (2010). Music-evoked nostalgia: affect, memory, and personality. Emotion, 10(3), 390-403.

Batcho, K. I. (1995). Nostalgia: a psychological perspective. Perceptual and Motor Skills, 80(1), 131-143.

Batcho, K. I. (1998). Personal nostalgia, world view, memory, and emotionality. Perceptual and Motor Skills, 87(1), 411-432.

Batcho, K. I. (2007). Nostalgia and the emotional tone and content of song lyrics. The American Journal of Psychology, 120(3), 361-381.

Batcho, K. I., DaRin, M. L., Nave, A. M. et Yaworsky, R. R. (2008). Nostalgia and identity in song lyrics. Psychology of Aesthetics, Creativity, and the Arts, 2(4), 236-244.

Belk, R. W. (1990). The role of possessions in constructing and maintaining a sense of past. Dans M. E. Goldberg (dir.), Advances in Consumer Research (vol. 17, p. 669-676). Provo, UT: Association for Consumer Research.

Belk, R. W. (1991). Possessions and the sense of past. Dans R. W. Belk (dir.), Highways and buyways: naturalistic research from the consumer behavior odyssey (p.114-130). Provo, UT: Association for Consumer Research.

Blackwell, R. et Stephan, T. (2004). Brands that rock. Hoboken, N.J.: John Wiley \& Sons.

Cattaneo, E. et Guerini, C. (2012). Assessing the revival potential of brands from the past: How relevant is nostalgia in retro branding strategies? Journal of Brand Management, 199(8), 680-687.

Cronbach, L. J. (1951). Coefficient alpha and the internal structure of tests. Psychometrika, 16(3), 297-334.

Davis, F. (1979). Yearning for yesterday: a sociology of nostalgia. New York, NY: Free Press.

Divard, R. et Robert-Demontrond, P. (1997). La nostalgie : un thème récent dans la recherche marketing. Recherche et Applications en Marketing, 12(4), 41-62.

Escalas, J. E. et Bettman, J. R. (2005). Self-construal, reference groups, and brand meaning. Journal of Consumer Research, 32(3), 378-389.

Godbole, M. B., Shehryar, O. et Hunt, D. M. (2006). Does nostalgia depend on valence of past experience? An empirical analysis of the discontinuity hypothesis. Dans C. Pechmann et L. Price (dir.), Advances in Consumer Research (vol. 33, p. 630). Duluth, MN: Association for Consumer Research.

Hallegatte, D. (2014). Retrobranding: selling the past. Dans G. Hunter et T. Steenburgh (dir.), AMA Winter Educators' Conference Proceedings s (vol. 25, p. K10-17). Orlando, FL: American Marketing Association.

Hallegatte, D. et Marticotte, F. (2014). Does Holbrook's nostalgia index measure nostalgia proneness? Dans G. Hunter et T. Steenburgh (dir.), AMA Winter Educators' Conference Proceedings (vol. 25, p. C84-90). Orlando, FL: American Marketing Association. 
Havlena, W. J. et Holak, S. L. (1991). "The good old days": observations on nostalgia and its role in consumer behavior. Dans R. H. Holman et M. R. Solomon (dir.), Advances in Consumer Research, (vol. 18, p. 323-329). Provo, UT: Association for Consumer Research.

Hepper, E. G., Ritchie, T. D., Sedikides, C. et Wildschut, T. (2012). Odyssey's end: lay conceptions of nostalgia reflect its original homeric meaning. Emotion, 12(1), 102-119.

Hepper, E. G., Ritchie, T. D., Sedikides, C. et Wildschut, T. (2010). A prototype analysis of nostalgia. 11th Annual Meeting of the Society for Personality and Social Psychology. Las Vegas, NV.

Herche, J. et Engelland, B. (1996). Reversed-polarity items and scale unidimensionality. Journal of the Academy of Marketing Science, 24(4), 366-374.

Hirschman, Elizabeth C., et Morris B. Holbrook. 1982. « Hedonic Consumption: Emerging Concepts, Methods and Propositions ». Journal of Marketing, vol. 46(3), 92-101.

Hofer, J. (1688). Medical dissertation on nostalgia by Johannes Hofer, 1688 (traduit du latin par Carolyn Kiser Anspach, 1934). Bulletin of the History of Medicine, 2(1), 376-391.

Holak, S. L., et Havlena, W. J. (1992). Nostalgia: an exploratory study of themes and emotions in the nostalgic experience. Dans J. F. Sherry et B. Sternthal (dir.), Advances in Consumer Research, (vol. 19, p. 380-387). Provo, UT: Association for Consumer Research.

Holbrook, M. B. (1993). Nostalgia and consumption preferences: some emerging patterns of consumer tastes. Journal of Consumer Research, 20(2), 245-256.

Holbrook, M. B. (1994). Nostalgia proneness and consumer tastes. Dans J. A. Howard (dir.), Buyer behavior in marketing strategy, (2e ed., p. 348-364). Englewood Cliffs, NJ: Prentice-Hall.

Holbrook, M. B. et Schindler, R. M. (1989). Some exploratory findings on the development of musical tastes. Journal of Consumer Research, 16(1), 119-124.

Holbrook, M. B. et Schindler, R. M. (1991). Echoes of the dear departed past: some work in progress on nostalgia. Dans R. H. Holman et M. R. Solomon (dir.), Advances in Consumer Research, (vol. 18, p. 330-333). Provo, UT: Association for Consumer Research.

Holbrook, M. B. et Schindler, R. M. (1994). Age, sex, and attitude toward the past as predictors of consumers' aesthetic tastes for cultural products. Journal of Marketing Research, 31(3), 412-422.

Holbrook, M. B. et Schindler, R. M. (1996). Market segmentation based on age and attitude Toward the past: concepts, methods, and findings concerning nostalgic influences on customer tastes. Journal of Business Research, 37(1), 27-39.

Iyer, A. et Jetten, J. (2011). What's left behind: identity continuity moderates the effect of nostalgia on well-being and life choices. Journal of Personality and Social Psychology, 101(1), 94-108.

Janataa, P., Tomic, S. T. et Rakowskia, S. K. (2007). Characterisation of music-evoked autobiographical memories. Memory, 15(8). 845-860.

Juhl, J., Routledge, C., Arndt, J., Sedikides, C. et Wildschut, T. (2010). Fighting the future with the past: nostalgia buffers existential threat. Journal of Research in Personality, 44(3), 309-314.

Juslin, P. N., Liljeström, S., Västfjäll, D., Barradas, G. et Silva, A. (2008). An experience sampling study of emotional reactions to music: listener, music, and situation. Emotion, 2008(5), 668-683.

Kessous, A., Roux, E. et Chandon, J.-L. (2015). Consumer-brand relationships: a contrast of nostalgic and non-nostalgic brands. Psychology \& Marketing, 32(2), 187-202.

Lambert-Pandraud, R. et Laurent, G. (2010). Why do older consumers buy older brands? The role of attachment and declining innovativeness. Journal of Marketing, 74(5), 104-121.

Leboe, J. P. et Ansons, T. L. (2006). On misattributing good remembering to a happy past: an investigation into the cognitive roots of nostalgia. Emotion, 6(4), 596-610.

Loveland, K. E., Smeesters, D. et Mandel, N. (2009). Still preoccupied with 1985: the effect of imagined interaction on preference for nostalgic products. Dans M. C. Campbell, J. J. Inman et R. Pieters (dir.), Advances in Consumer Research, (vol. 36, p. 64-65). Duluth, MN: Association for Consumer Research.

Loveland, K. E., Smeesters, D. et Mandel, N. (2010). Still preoccupied with 1995: the need to belong and preference for nostalgic products. Journal of Consumer Research, 37(3) 393-408.

Marchegiani, C. et Ian Phau. (2011). The value of historical nostalgia for marketing management. Marketing Intelligence \& Planning, 29(2), 108-122. 
McKechnie, G. E. (1974). ERI manual: environmental response inventory. Palo Alto, CA: Consulting Psychologists Press.

McKechnie, G. E. (1977). The environmental response inventory in application. Environment and Behavior, 9(2), 255-276.

Merchant, A., Ford, J. B. et Rose, G. (2011). How personal nostalgia influences giving to charity. Journal of Business Research, 64(6), 610-616.

Muehling, D. D. et Pascal, V. J. (2011). An empirical investigation of the differential effects of personal, historical, and nonnostalgic advertising on consumer responses. Journal of Advertising, 4092), 107-122.

Muehling, D. D. et Pascal, V. J. (2012). An Involvement Explanation for Nostalgia Advertising Effects. Journal of Promotion Management, 18(1), 100-118.

Muehling, D. D., Sprott, D. E. et Sultan, A. J. (2014). Exploring the boundaries of nostalgic advertising effects: a consideration of childhood brand exposure and attachment on consumers' responses to nostalgia-themed advertisements. Journal of Advertising, 43(1), 73-84.

Nawas, M. M. et Platt, J. J. (1965). A future-oriented theory of nostalgia. Journal of Individual Psychology, 21(1), 51-57.

Orth, U. R. et Gal, S. (2012). Nostalgic brands as mood boosters. Journal of Brand Management, 19(8), 666-679.

Park, C. W., MacInnis, D. J., Priester, J., Eisingerich, A. B. et Iacobucci, D. (2010). Brand attachment and brand attitude strength: conceptual and empirical differentiation of two critical brand equity drivers. Journal of Marketing, 74(6), 1-17.

Reisenwitz, T., Iyer, R., Kuhlmeier, D. B. et Eastman, J. K. (2007). The elderly's internet usage: an updated look. Journal of Consumer Marketing, 24(7), 406-418.

Reisenwitz, T. H., Iyer, R. et Cutler, B. (2004). Nostalgia advertising and the influence of nostalgia proneness. Marketing Management Journal, 14(2), 55-66.

Reynolds, S. (2011). Retromania: pop culture's addiction to its own past. New York, NY: Faber and Faber.

Rindfleisch, A., Freeman, D. et Burroughs, J. E. (2000). Nostalgia, materialism, and product preferences: an initial inquiry. Dans S. J. Hoch et R. J. Meyer (dir.), Advances in Consumer Research (vol. 27, p. 36-41). Provo, UT: Association for Consumer Research.

Rindfleisch, A. et Sprott, D. E. (2000). Moving forward on looking backward: advancing theory and practice in nostalgia. Dans S. J. Hoch et R. J. Meyer (dir.), Advances in Consumer Research, (vol. 27, p. 34-35). Provo, UT: Association for Consumer Research.

Routledge, C., Arndt, J., Sedikides, C. et Wildschut, T. (2008). A blast from the past: the terror management function of nostalgia. Journal of Experimental Social Psychology, 44(1), 132-140.

Routledge, C., Arndt, J., Wildschut, T., Sedikides, C., Hart, C. M., Juhl, J., Vingerhoets, Ad J. J. M. et Schlotz, W. (2011). The past makes the present meaningful: nostalgia as an existential resource. Journal of Personality and Social Psychology, 101(3), 638-652.

Schindler, R. M. et Holbrook, M. B. (2003). Nostalgia for early experience as a determinant of consumer preferences. Psychology \& Marketing, 20(4), 275-302

Sedikides, C., Wildschut, T., Arndt, J. et Routledge, C. (2008). Nostalgia: past, present, and future. Current Directions in Psychological Science, 17(5), 304-307.

Sedikides, C., Wildschut, T. et Baden, D. (2004). Nostalgia: Conceptual Issues and Existential Functions. Dans J. Greenberg, S. L. Koole et T. Pyszczynski (dir.), Handbook of experimental existential psychology, (200-214). New York, NY: Guilford Press.

Shankar, A., Elliott, R. et Fitchett, J. A. (2009). Identity, consumption and narratives of socialization. Marketing Theory, 9(1), 75-94.

Shumway, D. R. (1999). Rock 'n' roll sound track and the production of nostalgia. Cinema Journal, 38(2), p. 36-51.

Sierra, J. J. et McQuitty, S. (2007). Attitudes and emotions as determinants of nostalgia purchases: an application of social identity theory. Journal of Marketing Theory \& Practice, 15(2), 99-112.

Spears, N. et Amos, C. (2012). Revisiting western time orientations. Journal of Consumer Behaviour, 11(3), 189-197.

Sprott, D., Czellar, S. et Spangenberg, E. (2009). The importance of a general measure of brand engagement on market behavior: development and validation of a scale. Journal of Marketing Research, 46(1), 92-104.

Steenkamp, J.-B. E., Frenkel ter Hofstede, M. et Wedel, M. (1999). A cross-national investigation into the Individual and national cultural antecedents of consumer innovativenes. Journal of Marketing, 63(2), 55-69.

Stern, B. B. (1992). Nostalgia in advertising text: romancing the past. Dans J. F. Sherry et B. Sternthal (dir.), Advances in Consumer Research (vol. 19, p. 388-389. Provo, UT: Association for Consumer Research. 
Taylor, S. M. et Konrad, V. A. (1980). Scaling dispositions toward the past. Environment and Behavior, 12(3), 283-307.

Turner, R. N., Wildschut, T. et Sedikides, C. (2012). Dropping the weight stigma: nostalgia improves attitudes toward persons who are overweight. Journal of Experimental Social Psychology, 48(1), 130-137.

Wildschut, T., Sedikides, C., Arndt, J. et Routledge, C. (2006). Nostalgia: content, triggers, functions. Journal of Personality and Social Psychology, 91(5), 975-993.

Wildschut, T., Sedikides, C., Routledge, C., Arndt, J. et Cordaro, F. (2010). Nostalgia as a repository of social connectedness: The role of attachment-related avoidance. Journal of Personality and Social Psychology, 98(4), 573-586.

Wood, S. (2010). The comfort food fallacy: avoiding old favorites in times of change. Journal of Consumer Research, 36(6), 950-963.

Zentner, M., Grandjean, D. et Scherer, K. R. (2008). Emotions evoked by the sound of music: Characterization, classification, and measurement. Emotion, 8, 494-521.

Zhao, G., Muehling, D. D. et Kareklas, I. (2014). Remembering the good old days: the moderating role of consumer affective state on the effectiveness of nostalgic advertising. Journal of Advertising, 43(3), 244-255.

Zhou, X., Sedikides, C., Wildschut, T. et Ding-Guo, G. (2008). Counteracting loneliness: on the restorative function of nostalgia. Psychological Science, 19(10), 1023-1029.

Zhou, X., Wildschut, T., Sedikides, C., Chen, X. et Vingerhoets, Ad J. J. M. (2012a). Heartwarming memories: nostalgia maintains physiological comfort. Emotion, 12(4), 678-684.

Zhou, X., Wildschut, T., Sedikides, C., Shi, K. et Feng, C. (2012b). Nostalgia: the gift that keeps on giving. Journal of Consumer Research, 39(1) 39-50.

Zimbardo, P. G. et Boyd, J. N. (1999). Putting time in perspective: a valid, reliable individual-differences metric. Journal of Personality and Social Psychology, 77(6), 1271-1288.

Zimmer, M. R., Little, S. K. et Griffiths, J. S. (1999). The impact of nostalgia proneness and need for uniqueness on consumer perceptions of historical branding strategies. Dans A. Menon et A. Sharma (dir.), American Marketing Association Winter Educators' Conference Proceedings (vol. 10, p. 259-267). Chicago, IL: American Marketing Association. 